\title{
Mobile Accident Notification
}

\author{
Mevlüt İnan *1, Rıdvan Saraçoğlu ${ }^{1}$
}

Accepted $3^{\text {rd }}$ September 2016

\begin{abstract}
Traffic accidents are one of the most important and serious problems, our country encounters. As the percentage of injured and casualties in traffic accidents are huge in numbers, it causes thousands of people injured or dead in a year. In the situation of coming by a traffic accident that we face commonly in daily life, generally people directly use their mobile phones and call the related units.

However, as the required, right and adequate information is not given to these related units, the cases may sometimes end with casualties. One of the most important reasons of casualties in car accidents is the wrong, senseless first aids performed to the victims of injuries by surrenders. In this study, the aim is to determine the exact location of the accident with its latitude and longitude that is determined automatically via the application present in the responsive citizen who tries to help the victims of the accident.
\end{abstract}

With this system named as "Mobile Accident Notification" the users are aimed to send accident notification in online or via sending message when there is no internet access with the help of mobile applications that process in Android Operating System. These accident data kept in remote server are monitored on web pages or Android mobile phones. In this thesis, a web-based, open-source accident filtration system was developed and the accidents were provided to be monitored by the authorized people in related units.

Keywords: Mobile accident, Accident notification, Mobile accident tracking, Android mobile accident statement.

\section{Introduction}

Preventing traffic accidents is a shared concern of the whole world. Since the ratio of deaths and injuries significantly high, thousands of people die and hundreds of thousands of people get injured in considerably high number of accidents in each year. When an accident occurs in daily life, the common practice is to call relevant units and institutions. However, since sometimes correct information is not given to relevant units, many lives are lost. Minutes after the accident occurs (the time from the injury until the hospital care) are crucial for resuscitating accident victims and reducing physical effects of the accident. Improving first aid services is anticipated to reduce casualties up to $20 \%$ [1]. Among the reasons of injuries in traffic accidents resulting in casualties and permanent disabilities are late arriving ambulances and series of unapprised interventions of bystanders to accident victims. When reasons of late arrivals of ambulances are examined, various causes listed below are observed [2].

- Distance of the accident scene to the location of ambulances

- Ambulances receiving wrong directions

- Ambulance drivers not being able to locate accidents due to lack of knowledge of exact latitudes and longitudes of accidents.

- Directing ambulances to false locations (pranks etc.)

- Deploying un-operational ambulances from faraway centers to the accident scene, rather than nearest ones, due to lack of communication

- Inadequate number of ambulances when the accident

\footnotetext{
${ }^{1}$ Başkale Vocational High School, Yüzüncü Yll University, Campus, 65080, Van/Turkey

* Corresponding Author: Email: mevlutinan@yyu.edu.tr

Note: This paper has been presented at the $3^{\text {rd }}$ International Conference on Advanced Technology \& Sciences (ICAT'16) held in Konya (Turkey), September 01-03, 2016.
}

\section{occurred \\ ○ Traffic jams}

- Ambulances not being able to pull up close to accident scenes due to irregularly parked cars on streets and avenues.

The objective of this thesis is to avail responsive citizens to notify accidents with an application downloaded into their personal mobile phones and to directly locate the scene of the accident on the map by automatically retrieving latitude and longitude values of the informant. Additionally, by filling a form included with the application, appropriate number of ambulances and police will be diverted to the accident scene since the exact number of people injured will be learned or possibly life saving information will be given to healthcare professionals since the nature of the injuries will be identified. Further objectives of this thesis are grouped under general headlines below.

\section{Accident Notification And Detection Systems}

Accident notification systems are usually the process of calling the phone numbers of related units and informing about an accident. With this notification method generally people either call the police and request other units to be dispatched or call the emergency services to request an ambulance to be sent to the scene of the accident.

In case of accident detection systems, usually the detection is realized by reading the data from sensors attached to vehicles. Another method is a study conducted by Amin et al[3] where data from GPSs of smart phones are read in 0.1 second intervals and the location of the vehicle is located by map matching algorithms and then the previous speed values collected in 0.1 second intervals are subtracted from the subsequent speed values where the difference is then compared to the threshold values to determine if an accident occurred or not. 
Additionally there are other solutions such as detecting accidents without interaction with Electronic Control Units, false positive preventions and WreckWatch client-server architecture. One final distinctive study is done by placing noise and image recording cameras on junctions and start recording exactly when the accident occurs by recognizing the noises from the cars involved in an accident and by not recording continuously but only when the accident took place [4].

\section{System Flow Diagram}

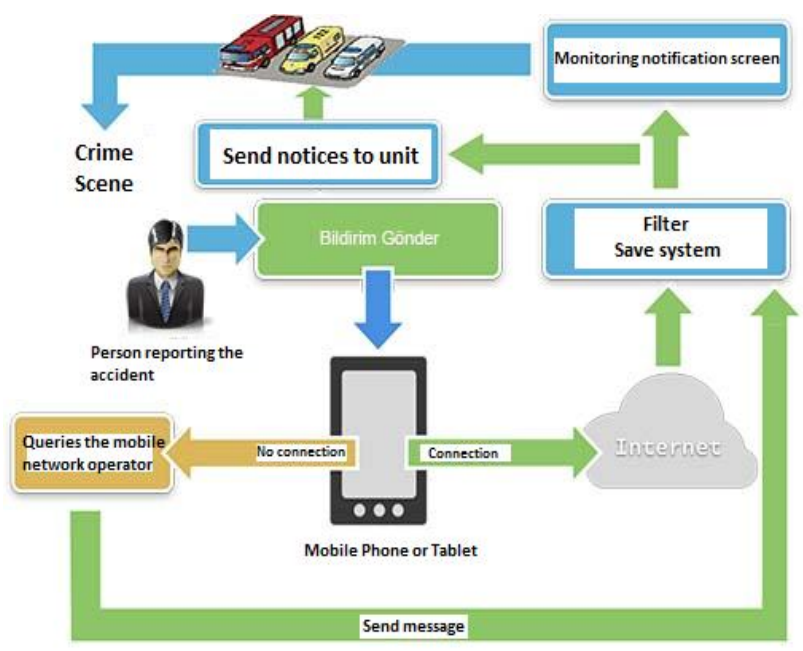

Figure 1. System flow chart

\section{Accident notification by the accident informant as the user}

\subsection{Notification via text messaging}

It is a situation where there are no internet connections and the user gives the notification without filling the form (GPS must be active). When the user sends a notification via text message, an SMS filter application installed smart phone receives this message. Application divides this SMS into meaningful pieces and sends it to the database of the system through the internet. In case where there is no internet connection, the application writes it to its own local and safe keeps it. When there is an internet connection, it sends it expeditiously.

\subsection{Fast Notification}

User prefers this method if there is internet connection and when he/she does not want to enter too much information. When the user clicks on fast notification (GPS must be active), a record of the accident is created in the database immediately. The other information on the form is updated with the data entered by the user. Since the location of the accident and the location where the user filled the form may significantly differ, a record is immediately created when fast notification is clicked. For that reason the record is created immediately after the user clicked on the fast notification; other information can be updated at a later time.

\subsection{Detailed Notification}

The user prefers this method if there is internet connection and if he/she is willing to give detailed information such as a photograph of the accident scene, license plates of vehicles, number of vehicles involved, estimated distance of the accident, etc. When the user clicks to the detailed notification (GPS must be active), a record of the accident is created on the system database. Other information is updated by the user entering appropriate data. When detailed notification is clicked, the notification is generated. The reason for creating an immediate record is explained in Fast Notification section.

\section{Filtering Notifications}

When an accident occurs, many notifications are sent by users. However since these notifications are for the same accident, they have to be filtered and recorded under one singular accident. For that purpose several constraints should be defined. These constraints can be listed as follows;

- Diameter

Indicates the required diameter for notifications sent by different users to be perceived as belonging to the same accident. It is a parameter used to add all informants under the same category.

$\circ$ Estimated distance

This is a criteria put forth to receive data from the nearest point to the accident among many different notifications by taking the estimated distance of the informant to the accident scene.

\section{Sending Related Accident Message To Relevant Unit Workers}

This is the process of sending messages according to the notifications of various informants to the closest Police Headquarters, Health Institutions and Fire Department workers.

\section{M- Accident Notification Application}

With this application; it is targeted to create awareness among end-users by involving latest technologies to intervene accidents in a timely manner. This study came forth with the idea of ensuring fast and productive interference to accidents and with minimizing the response period during when accidents occur until first aid services reached to victims, is developed by using many integrated web and mobile technologies altogether.

In the thesis, two of them mobile, one of them web based, three different applications are developed. A single database is used for every each application. The application is consisted of three modules. These are; SMS Filtering module, Notification Sending module and administrator follow-up module. Detailed explanations and screen shots from these modules will be given in the upcoming sections.

\section{Conclusion}

One of the major concerns of our country as well as the world is traffic accidents. In the name of preventing these accidents which result in hundreds of thousands of casualties each year and cause many of them left with permanent disabilities, science people and researches have conducted many widespread studies. In the light of these studies, they have proposed several accident detection and accident tracking systems underlying how these accidents can be prevented. In one of those studies, a three-step protection method has been offered by Kraus et al[5]. The first of these protection methods is the detection of causes of accidents and taking necessary protections against them; the second method is things that need to be done when an accident occurs and measures that need to be taken to reduce severe effects of accidents; and the final method is what needs to be done after accidents occur and necessary precautions to minimize effects of injuries[5]. 
Taking inspiration from what needs to be done when an accident occurs, as pointed out in the second method of the study by Kraus et al above, and many other similar studies, actions to be taken in order to minimize the time from the moment the accident occurs until the first intervention to the accident victim (the golden hour) and to treat accident victims in the shortest possible time with the help of the support of a mobile application has been tried to be conveyed in this thesis. With this study, automatically obtaining latitudes and longitudes of exact locations of user/users who have witnessed the accident through GPS, either via the internet connection or when there are no internet connections, via SMS text messaging method has been achieved. With numbers of people injured as well as other pertinent data entered in the form, dispatching both adequate number of ambulances and diverting relevant police units to the accident scene have been targeted. Depending on the nature of injuries, determining and transferring probably life saving information to healthcare professionals has been proposed. At the present time when an accident occurs, among reasons of late arrivals of relevant units to the accident scene are; receiving wrong directions or not being able to find the correct address. With this application, actions such as; direct determination of the exact location of the accident on the map by taking the information of the closets users was targeted. In summary, with this thesis actions such as; contacting emergency services of nearest hospitals, contacting nearest Police Headquarters, totally eliminating the risk of giving wrong directions to ambulances, dispatching ambulances to the correct location by extracting exact latitudes and longitudes of accident scenes on the map, by collecting data from the informant such as his/her exact latitude and longitude, number of vehicles involved, number of injured, and the nature of injuries, dispatching adequate number of ambulances with appropriate equipment, when tens, sometimes hundreds of people inform an accident, giving only the notification of the relevant accident will be taken. When this mobile application is used, it is our belief that the time passed from the moment the accident occurs until the first intervention to accident victims will be minimized, adequate number of equipment and vehicles will be dispatched to the accident scene, assistance will arrive on the shortest time possible by utilizing maps, closest relevant units will be dispatched to the location and many similar advantages will be provided. No such study has been conducted ever before. Therefore a study like this is believed to immensely contribute to the literature as well.

\section{References}

[1] K. Sangeetha, P. Archana, M. Ramya, and P. Ramya, "Automatic ambulance rescue with intelligent traffic light system, “, IOSR Journal of Engineering(IOSRJEN), vol. 4, pp. 53-57, 2014.

[2] Ch. R. Keerthi, G. Shanmukh, and R. Sivaram, "Various accident detection technologies and recovery systems with victim analysis, ", International Journal of Advanced Trends in Computer Science and Engineering (IJATCSE), vol. 2, pp. 07-12, 2013.

[3] MD. S. Amin, M. A. S. Bhuiyan, M. B. I. Reaz, and S. S. Nasir, "GPS and map matching based vehicle accident detection system, “ IEEE Student Conference on Research and Development (SCOReD), Putrajaya, Malaysia, Dec. 2013.

[4] P. J. Lagassey, "Advanced automobile accident detection, data recordation and reporting system," U.S. Patent 7348 895, 2008.

[5] J. Kraus, C. Peek-Asa, D. Vimalachandra, "Injury control: The Public Health Approach, " Maxcy- Rosenau-Last, Public Health\&Preventive Medicine, Wallace Robert B., MD, Msc, vol.2, pp. 1209-1210, 1998. 